\title{
A Educação Interprofissional e a prática compartilhada em programas de residência multiprofissional em Saúde ${ }^{*}$
}

Isis Alexandrina Casanova ${ }^{(a)}$ Nildo Alves Batista ${ }^{(b)}$ Lídia Ruiz Moreno(c)

Casanova IA, Batista NA, Moreno LR. Interprofessional Education and shared practice in multiprofessional health residency programs. Interface (Botucatu). 2018; 22(Supl.1):1325-37.

The aim of this paper is to present the principles, conceptions, and practices of Interprofessional Education that underlie multiprofessional health residency programs (PRMS), with emphasis on shared practice, in institutions of higher education in the state of São Paulo, Brazil. The researchers conducted document analysis of the programs' pedagogical projects (PP) and administered a Likerttype scale and open-ended questions to 76 residents of 13 PRMS. Thematic analysis of the PP showed that emphasis was given to the team in clinical cases and care plans; patient-centeredness and coordination between teaching and service. The results of the Likert-type scale showed that residents were satisfied with education for collaborative practice. From the point of view of the residents, the PRMS helped expand and enhance health outcomes, favoring the patient-centered care, the identification of health needs, and shared practices and procedures. In conclusion, PRMS are important to the Brazilian National Health System (SUS).

Keywords: Human resources education. Internship and residency. Interprofessional education.
$\mathrm{O}$ artigo tem por objetivo analisar os princípios, concepções e práticas da Educação Interprofissional, com ênfase na prática compartilhada em programas de residência multiprofissional em Saúde (PRMS) nas instituições de ensino superior (IES) do Estado de São Paulo, Brasil. Foi realizada análise documental dos projetos pedagógicos (PP), aplicação de instrumento tipo Likert e perguntas abertas a 76 residentes de 13 PRMS. A análise temática dos PP mostra ênfase na responsabilização da equipe perante os casos clínicos e planos de cuidado; centralização nas necessidades do paciente e articulação entre ensinoserviço. Os resultados do instrumento Likert evidenciam satisfação dos residentes com a formação para prática colaborativa. Na perspectiva dos residentes, os PRMS ampliam e melhoram os resultados em saúde, favorecem a atuação centrada no paciente, a identificação das necessidades de saúde e o compartilhamento de práticas e procedimentos. Dessa maneira, os PRMS são importantes no cenário de consolidação do Sistema Único de Saúde (SUS).

Palavras-chave: Formação de recursos humanos. Internato e residência. Educação interprofissional.
Este artigo é resultado do Projeto "A Educação Interprofissional na Residência Multiprofissional em Saúde no Estado de São Paulo", financiado pela Capes, Edital 024/2010 - Pró-Ensino na Saúde (AUXPE n 1605/2011).

(a) Fonoaudióloga. São Paulo, SP, Brasil. isiscasanova@hotmail.com

$(b, c)$ Centro do Desenvolvimento do Ensino Superior em Saúde (Cedess), Universidade Federal de São Paulo. São Paulo, SP, Brasil. nbatista@unifesp.br; lidia.ruiz@unifesp.br 


\section{Introdução}

Existe uma demanda no trabalho em saúde que transcende os fazeres individualizados de cada profissão e projeta a valorização da equipe, na qual o profissional não abre mão da sua especificidade, mas valoriza o trabalho cooperativo em ações direcionadas à população.

Com a finalidade de reorientar os serviços e os atendimentos de acordo com as necessidades de saúde, principalmente no que se refere à Atenção Básica, surge a Residência Multiprofissional em Saúde (RMS), promulgada a partir da Lei $\mathrm{n}^{\circ} 11.129$ de $2005^{1}$ e orientada pelos princípios e diretrizes do Sistema Único de Saúde (SUS).

A RMS contribui na formação e na qualificação do profissional na saúde, tem como proposta o comprometimento com o cuidado, principalmente no que tange à integralidade, e propicia mudanças necessárias no modelo técnico-assistencial.

A residência aprofunda os debates teórico-práticos e permite a construção de novos saberes entre as diferentes categorias profissionais da saúde².

Nesse contexto de valorização do trabalho de equipe, insere-se a educação interprofissional (EIP). A origem desse termo consta no relatório da Organização Mundial de Saúde (OMS) de 1988. Nesse documento, foram evidenciadas experiências de alguns países acerca do aprendizado em conjunto entre diferentes profissões nos seus locais de trabalho. A OMS ${ }^{3}$ define a EIP como: "[...] o aprendizado que ocorre quando estudantes de duas ou mais profissões aprendem sobre os outros, com os outros e entre si para possibilitar a colaboração eficaz e melhorar os resultados de saúde" (p. 7).

Para atingir a prática colaborativa ${ }^{3}$, os profissionais devem aprender a trabalhar como membros efetivos das equipes interprofissionais. O Centro para o Avanço e Desenvolvimento da EIP do Reino Unido, originado em 1987, (The Center for Advancement of IPE - Caipe) ${ }^{4}$ define a EIP como um processo de ensino e aprendizagem que promove a colaboração no trabalho e melhora a qualidade do atendimento a partir da prática colaborativa.

Essas ações transcendem um atendimento em equipe e proporcionam uma maior organização em torno das necessidades de saúde local. Todavia, os profissionais que participam de uma prática colaborativa necessitam de modelos de governança claros e objetivos e de protocolos de atendimento que favoreçam a interação para alcançar os objetivos propostos neste 4 .

O Centro Nacional de Saúde Interprofissional Colaborativo Canadense (Canadian Interprofissional Health Collaborative $-\mathrm{CIHC})^{5}$ reconhece que a EIP e a prática colaborativa favorece melhores resultados aos usuários a partir do desenvolvimento de habilidades, conhecimentos e valores necessários para o trabalho em equipe.

No Brasil| ${ }^{6}$, existem iniciativas que têm propiciado o desenvolvimento de propostas e pesquisas de sucesso, mediante a reorganização do modus operandi de suas equipes. Essas iniciativas se encontram na área da Atenção Básica da saúde mental e nos hospitais públicos e possui como finalidade o favorecimento da colaboração interprofissional.

$\mathrm{Na}$ experiência relatada por Rossit et al. ${ }^{7}$, da Universidade Federal de São Paulo (Unifesp), campus Baixada Santista, os cursos de graduação são integrados em um currículo interprofissional. O projeto pedagógico orienta a formação de profissionais de saúde para o trabalho de equipe interprofissional, com ênfase na integralidade do cuidado. Um aspecto central dessa experiência foi a composição intencional de grupos dos estudantes dos seis cursos oferecidos (psicologia, educação física, serviço social, nutrição, terapia ocupacional) desde 2006.

$\mathrm{Na}$ graduação dessa instituição, a experiência interprofissional estimulou a criação de outras propostas formativas na pós-graduação lato sensu e stricto sensu, com foco na EIP. Com base nesses princípios, foi criado o Programa de Residência Integrada Multiprofissional em Atenção à Saúde (Primas) com os cursos de enfermagem, nutrição, terapia ocupacional, farmácia, fisioterapia, psicologia e de serviço social, bem como o Mestrado em Ciências da Saúde.

De acordo com Barr ${ }^{8}$, as propostas de EIP encontram resistências por parte dos docentes das universidades. O autor afirma que, para superá-las, são necessárias novas perspectivas nos processos de: ensino-aprendizagem, formação docente, suporte institucional, recursos financeiros e valorização do corpo docente. 
Para Batista e Batista9 ${ }^{9}$ a possibilidade de introduzir a EIP nos currículos das instituições requer habilidade, preparação para lidar com as adversidades e a responsabilidade dos participantes. Alguns atributos são necessários nesse processo: a experiência prévia em trabalho interprofissional, métodos interativos de aprendizagem, conhecimento de dinâmicas de grupo, confiança em trabalhar com grupos interprofissionais e uma atitude flexível.

A EIP pode representar oportunidades de formação conjunta para o desenvolvimento de aprendizagens compartilhadas, a fim de melhorar a qualidade da assistência ao paciente.

Com ênfase na prática colaborativa, a presente pesquisa teve por objetivo a análise dos princípios, das concepções e das práticas da EIP nos PRMS, de acordo com a perspectiva dos residentes, e no PP de IES do Estado de São Paulo.

\section{Métodos}

Esse estudo consistiu em uma pesquisa quali-quantitativa ${ }^{10}$ de caráter exploratório descritivo ${ }^{11}$. Como critério de inclusão, consideram-se os PRMS oferecidos no Estado de São Paulo e em funcionamento no ano de 2014, cujos residentes deveriam estar no segundo ano (R2) e pertencer, no mínimo, a três profissões de diferentes áreas da saúde.

De acordo com esses critérios, identificou-se, no portal do Ministério de Educação e Cultura-MEC ${ }^{12}$, um total de 14 IES que foram convidadas a participar da pesquisa por meio da Plataforma Brasil. Apenas três IES aceitaram o convite e uma delas não pode ser incluída na pesquisa, pois os residentes estavam no primeiro ano.

Nas duas IES incluídas na pesquisa, funcionam 23 PRMS, das quais três são uniprofissionais, três possuem residentes ainda no primeiro ano (R1) e quatro não concordaram em participar da pesquisa. No total, foram 13 programas pesquisados: oito programas da Universidade Federal de São Paulo (Unifesp), em que sete foram do campus São Paulo e um do campus Baixada Santista; e cinco programas da Faculdade de Medicina de Marília (Famema). Do total de 121 residentes (R2), 76 (63\%) participaram da pesquisa. A coleta de dados foi realizada entre dezembro de 2014 e março de 2015.

Foi realizada, na modalidade temática ${ }^{13}$, uma pesquisa documental dos 13 PP (identificados por número arábico de um a 13) oriunda dos sites dos PRMS. Os dados obtidos foram agrupados em unidades de contextos (UC) e em unidades de registros (UR) que se constituem no menor recorte de ordem semântica advinda do texto, representada por uma palavra, frase ou oração, das quais foram apreendidas as categorias.

Para explorar os PP, uma pergunta norteadora foi elaborada: O PRMS estabelece, na prática colaborativa dos residentes, a responsabilização pelo cuidado com as metas e os objetivos comuns, de acordo com as necessidades da população?

A pesquisa de campo consistiu na aplicação de um instrumento tipo likert, desenvolvido por Perego ${ }^{14,15}$. As asserções relacionadas à prática colaborativa foram: o desenvolvimento de competências para a prática colaborativa (A1); a interação entre as diferentes profissões (A3); a integração dos profissionais de saúde (A7); a atuação integrada entre os profissionais (A9); a força de trabalho colaborativo, o apoio institucional, a cultura de trabalho e o ambiente (A10); a visão ampliada da saúde (A14); e a satisfação do paciente durante o atendimento e melhores resultados em saúde (Figura 1). As respostas do instrumento tipo likert foram submetidas a uma análise estatística com o teste de confiabilidade de 0,84.

Para análise dos dados, tomou-se por base as pontuações das assertivas e das dimensões que poderiam variar de 1 a 4 . A análise do instrumento likert se baseou em três intervalos de pontuação: de 1 a 1,99 - zona de perigo: a percepção é negativa e as mudanças de curto prazo devem ser tomadas; de 2,00 a 2,99 - zona de alerta: a percepção revela aspectos a serem melhorados e exige medidas a médio prazo; e de 3,00 a 4,00 - zona de conforto: a percepção é positiva e pode ser potencializada ${ }^{16}$.

A coleta também compreendeu a aplicação de um questionário para a caracterização do perfil dos participantes: idade, sexo, profissão e egresso de instituição de ensino superior (pública ou privada). 
Quanto à caracterização da amostra, essa foi constituída de 21 enfermeiros, 12 fisioterapeutas, 11 psicólogos, dez assistentes sociais, dez nutricionistas, cinco fonoaudiólogos, quatro terapeutas ocupacionais, dois farmacêuticos e um educador físico. Dos participantes, 74 eram do sexo feminino e dois do sexo masculino. Quanto à faixa etária, 46 residentes tinham menos de 26 anos de idade, 25 residentes estavam na faixa etária entre 26 e 31 anos e cinco residentes tinham 31 anos ou mais. Em relação à natureza jurídica das IES de graduação, 45 participantes eram de instituições públicas e 31, de instituições privadas.

Os R2 também responderam a uma questão aberta sobre a formação durante a RMS, com ênfase na prática colaborativa. A pergunta realizada foi: "Em sua opinião, a RMS prepara o profissional de saúde para uma prática colaborativa? Comente." As respostas foram submetidas à análise temática de acordo com Bardin ${ }^{13}$.

Esse trabalho é parte integrante de uma tese de doutorado. A pesquisa está em consonância com os preceitos éticos, firmados na Resolução n 466/2012 e foi aprovada pelo conselho de ética.

\section{Construção de resultados}

\section{Educação interprofissional e a prática colaborativa nos PRMS: uma análise a partir dos PP}

A primeira categoria apreendida nos documentos - "Responsabilização na condução dos casos clínicos e dos planos de cuidado" - presente nos PP de números 1, 3, 4 e 5 ressalta, como uma das principais ações da força de trabalho em saúde, a atuação conjunta dos profissionais de acordo com a necessidade da população.

[...] análise de informações e da elaboração de planos de cuidado (individual e coletivo) e de organização do trabalho em saúde [...] o residente é responsável pela identificação de necessidades de saúde individuais e coletivas e da elaboração, execução e avaliação do plano de cuidados para os problemas identificados. (PP4, PP5)

Tem a responsabilidade principal pela condução do caso. [...] aumentar a capacidade resolutiva de problemas de saúde pela equipe, [...] que pode se efetivar através de ações conjuntas: discussões de casos, intervenções junto às famílias e comunidades, proposição de atividades específicas e construção de projetos terapêuticos singulares. [...] qualificando e ampliando a clínica na equipe. (PP1, PP3)

A centralização nas necessidades do paciente com melhora dos resultados em saúde corresponde à segunda categoria apreendida e remete a uma prática com foco no paciente, no planejamento das ações e na qualidade do atendimento, a fim de estabelecer a confiança e a colaboração entre os profissionais. Essas ações são destacadas nos PP de 1 a 3.

[...] Levarão em consideração as dimensões subjetiva e social de cada pessoa e de cada família, que tornam singular a experiência de adoecimento, visando uma melhor compreensão sobre as condições de vida [...] voltados para a melhoria da qualidade de vida [...] (PP1, PP2, PP3)

[...] estabelecimento de objetivos, metas e indicadores relativos ao cuidado e o alinhamento de diretrizes clínicas e/ou protocolos, baseada em uma visão clínica ampliada e centrada nas necessidades do paciente. (PP1, PP2, PP3)

A maioria dos documentos analisados não menciona o termo EIP; contudo, explicita conceitos e práticas que se aproximam da EIP e faz referência à importância do auxílio na formação integrada que corresponda às necessidades locais. 
Ademais, o desenvolvimento da capacidade do trabalho de equipe é enfatizado com o objetivo de buscar a identificação, a aproximação e o conhecimento por meio de vivências em diferentes cenários de aprendizagem que visem à integração entre a equipe concomitante, a articulação teórica e a prática, de acordo com Aguilar-da Silva et al. ${ }^{17}$.

É possível se aproximar dos problemas de saúde locais com o uso de abordagens diferenciadas no processo saúde/doença, a partir do desenvolvimento de competências comuns, da aprendizagem compartilhada, da capacidade de resolução de problemas e do respeito mútuo quando se parte da colaboração ${ }^{17}$.

"A associação do conhecimento acadêmico com a prática do serviço" - a última categoria - retrata o benefício que o trabalho da equipe conquista quando concilia a teoria e a prática profissional nos serviços de saúde e carrega consigo a reflexão crítica no atendimento.

Profissionais preparados para o trabalho em equipe interprofissional, para atuar em todos os níveis de atenção à saúde, com consciência crítica reflexiva sobre o processo saúdeadoecimento-cuidado e capacidade para fazer a intermediação entre o conhecimento acadêmico e as práticas nos serviços de saúde, junto a indivíduos, famílias e redes sociais. (PP8)

Embora o conjunto dos PP aproxime os conceitos relacionados à EIP, existem ênfases e detalhamentos diferentes nos documentos analisados. O PP 8 é o único que apresenta, explicitamente, o conceito da EIP. A implementação da EIP e da prática colaborativa favorece um trabalho coeso, com o envolvimento de todos; proporciona a satisfação e a sinergia entre os membros da equipe; e promove o reconhecimento e o respeito de cada cultura profissional ${ }^{17}$.

Os demais PP $(6,7,9,10,11,12$ e 13) preveem atividades teóricas e teórico-práticas, integradas e multidisciplinares. Todavia, não citam, especificamente, metas e objetivos comuns pertinentes à EIP. Contudo, por se tratar de programas inseridos no SUS, é possível que essa prática esteja presente nas ações das equipes no que se refere à prática ao cuidado.

Para Rossit et al.7, a EIP, vivenciada no processo de formação, permite que o futuro profissional se torne um indivíduo mais crítico e mais reflexivo, comprometido com as ações de saúde; com um olhar mais sensível ao contexto socioeconômico, familiar e cultural da população e do paciente; e mais integrado à equipe. Acredita-se que a prática colaborativa, por meio do planejamento, intervenção e avaliação das ações de saúde, permita aos residentes um maior aprendizado no coletivo e no trabalho de equipe com a integralidade do cuidado.

A EIP e a prática colaborativa maximizam as ações de saúde dos profissionais; favorecem seu desempenho quanto aos conhecimentos e habilidades desenvolvidas; e proporcionam atendimentos coordenados em situações cotidianas. Com relação à Atenção à Saúde, a prática colaborativa resulta na segurança e na satisfação dos pacientes quanto aos resultados em saúde, bem como no uso adequado dos recursos clínicos especializados. A EIP e a prática colaborativa não significam uma "cura", porém, quando praticadas, desenvolvem a força de trabalho em saúde de maneira participativa, capaz de gerar respostas efetivas e assistenciais ${ }^{3}$.

\section{Percepção dos residentes sobre o desenvolvimento de competências para práticas colaborativas}

As respostas dos residentes, relacionadas às asserções apresentadas no instrumento tipo likert acerca da prática colaborativa, estão na zona de conforto, o que denota a sua satisfação com o desenvolvimento de competências relacionadas a essa prática durante a RMS.

As asserções do instrumento se referem aos seguintes aspectos: 1) as práticas colaborativas permitem que os residentes compreendam a importância da atuação conjunta, o que é essencial para a segurança e para a saúde do paciente; 2 ) as práticas colaborativas envolvem o compartilhamento das competências dos residentes e potencializa as suas habilidades e os seus pontos fortes, com uma visão ampla da saúde e do bem-estar; 3) a satisfação e a percepção dos R2, em relação ao conjunto das asserções, refletem a média $(3,31)$, situada na zona de conforto. Esse dado demonstra que existe um consenso, por parte dos residentes, em relação à prática compartilhada na equipe. 


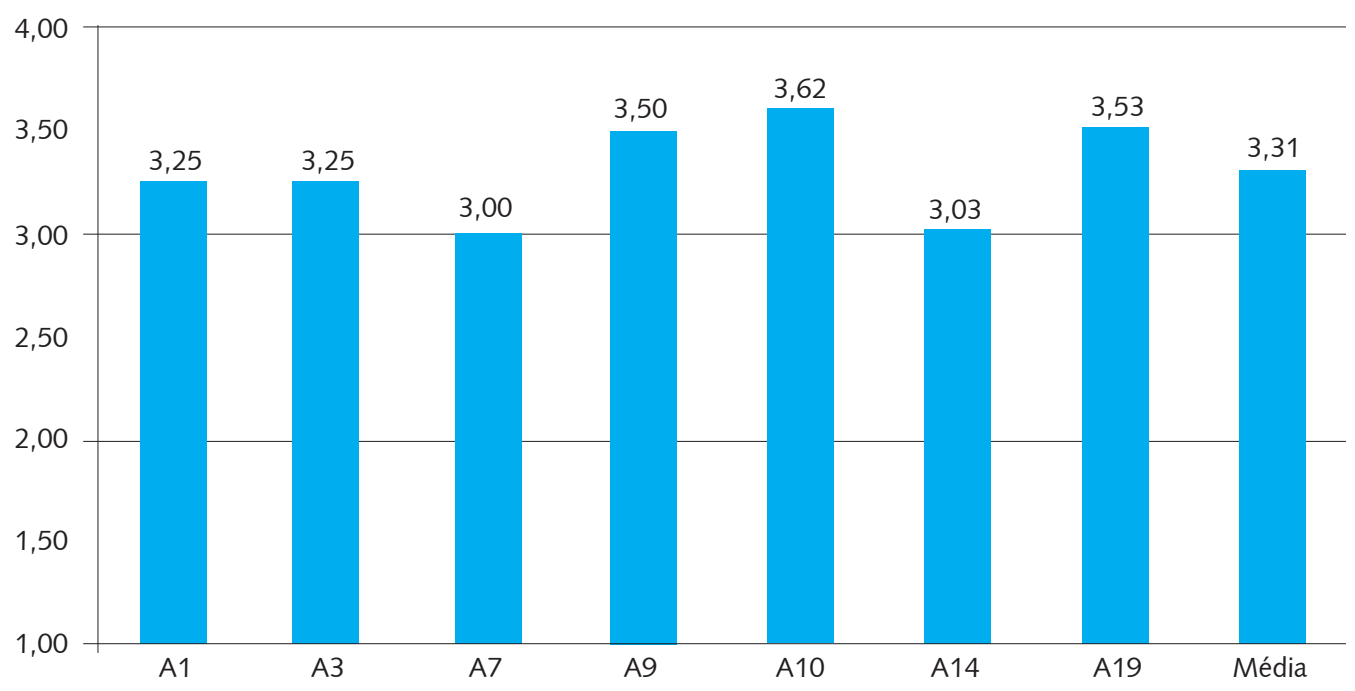

Figura 1. Valores das médias das asserções validadas pertencentes à análise da categoria "Desenvolvimento de práticas colaborativas" e seu perfil geral atitudinal.

Com a visão do trabalho em equipe, é possível a criação de novos espaços que se originam das práticas no território; das trocas de saberes, de experiências e de transformação, mediante a corresponsabilidade pelo projeto terapêutico, sem a existência de uma rivalidade corporativa, o que se torna um apoio necessário para a concretização do projeto, no qual existe o confronto da lógica do processo de formação e do trabalho tecnicista e hegemônico pautado na atuação coletiva e nas rotinas de cuidado com a saúde, aspectos relevantes destacados por Ceccim ${ }^{18}$.

D'Amour et al. ${ }^{19}$ ressaltam que as práticas colaborativas são caracterizadas pelo respeito mútuo e pela confiança. Os profissionais devem reconhecer as diferentes áreas da saúde como uma complementaridade dos saberes e das ações entre a equipe, dentro da perspectiva do cuidado integral do paciente.

\section{O olhar do residente: desafios e potencialidades da EIP e a prática colaborativa nos PRMS}

Os dados, obtidos das asserções do instrumento likert, evidenciam uma percepção positiva dos residentes quanto à prática colaborativa e à EIP. Todavia, a análise qualitativa das questões abertas permitiu aprofundar o entendimento sobre as potencialidades e desafios da EIP nos PRMS.

A primeira categoria, das respostas dissertativas dos residentes, demonstra o enfoque na prática colaborativa para a melhora dos resultados em saúde:

Através da integração da prática e dos saberes com outras áreas profissionais, torna-se mais fácil e mais eficaz o cuidado do paciente. Essa experiência é vivida na residência, tornando a prática mais colaborativa. (E17)

[...] a prática colaborativa é fruto de horas intensas de discussões [...] com os profissionais dos serviços e do atendimento do usuário [...]. (E43) 
Motta e Pacheco ${ }^{20}$ enfatizam que os PRMS desenvolvem a formação interprofissional; a prática colaborativa centrada no paciente; o cuidado integrado; e o entendimento de que, ao trabalhar de modo interprofissional, o residente assegura o tratamento consoante à realidade da comunidade, da família e do paciente.

A EIP prioriza a força de trabalho conjunto e a inserção em cenários de aprendizagem que visem à integração da teoria com a prática e do ensino com o serviço. Essa abordagem favorece uma nova maneira de ser, fazer, conhecer e conviver. Silva ${ }^{21}$ considera esses aspectos importantes. O trabalho de equipe permite uma prática mais propositiva e resolutiva à integralidade do cuidado.

De acordo com o autor ${ }^{21}$, é importante a criação de recursos cognitivos e afetivos, cujo objetivo é priorizar e identificar as necessidades de saúde da comunidade e o respeito pela sua autonomia. Desse modo, qualquer problema de saúde é compreendido e articulado no processo saúde-doença. Contudo, a prática colaborativa ainda é um desafio, conforme comentado por um residente: " [...] o que vivo na prática, ainda há muita resistência entre algumas áreas de saúde a entender e compartilhar conhecimentos específicos de cada categoria profissional, o que acaba prejudicando a sinergia da equipe e o próprio trabalho em equipe" (E42).

O desafio da EIP, na perspectiva do cuidado integral, recai na formação especializada que dificulta a visão das necessidades dos usuários. Silva ${ }^{21}$ explica que esse modelo fragmentado das práticas em saúde organizado na lógica do mercado nem sempre desenvolve competências para o trabalho de equipe interprofissional.

O autor ${ }^{22}$ ressalta que o conhecimento de um único profissional não é suficiente para atender às necessidades do usuário/família/comunidade e que, mediante a responsabilização em reconhecer a importância do cuidado, as ações em saúde tendem a motivar, consequentemente, o trabalho de equipe interprofissional.

Diante das diferentes profissões da saúde, os conflitos e dificuldades se relacionam entre si, na tentativa de manter o foco na comunidade, no usuário e na organização dos serviços. Cabe destacar que os autores Ellery et al. ${ }^{23}$ esclarecem que a EIP pode impedir que esses espaços de saúde se transformem em "campos de batalha" na busca, por parte dos profissionais, de reconhecimento, prestígio e poder, o que favoreceria a atuação da equipe.

Os residentes relatam a necessidade de uma definição conjunta de indicadores para avaliar a qualidade do serviço prestado pela equipe, na tentativa de melhorar o cuidado com o paciente.

[...] o trabalho é desenvolvido sem a clareza de indicadores para darem concretude ao cuidado prestado e parâmetros para avaliação da qualidade do serviço ofertado. (E12)

[...] faltam metas estipuladas para dar objetividade à atenção em saúde prestada, bem como foco para a equipe e parâmetro para avaliação da qualidade do serviço prestado. (E50)

A avaliação dos serviços contribui para a qualidade da assistência e para o desenvolvimento de políticas públicas. No que se refere às áreas de saúde e de educação, os formuladores de políticas devem promover, ao implementarem a EIP, mecanismos de avaliação que mensurem a qualidade do atendimento, uma vez que são raras as avaliações sobre a EIP, nos resultados relacionados à saúde e aos serviços prestados ${ }^{3}$.

A categoria "Atuação conjunta com centralidade no paciente" pode ser entendida como um processo, no qual se pretende que o atendimento do profissional suplante uma atuação restrita.

O meu exemplo de residência e aprendizagem [...] são as discussões multiprofissionais em que toda a equipe da unidade consegue conversar sobre o mesmo paciente e pensar em um plano mais amplo para sua recuperação e possível alta [...]. (E21)

Quando as visitas multiprofissionais são realizadas, os trabalhos e as discussões são feitas, abrese espaço para que cada profissional mostre o seu papel e a colaboração que pode oferecer no cuidado ao paciente. (E23) 
Os resultados, que se constroem durante o atendimento na RMS, reforçam a relevância da prática colaborativa entre os profissionais e propõem um novo cenário de atuação e de reorientação da equipe. Nesse sentido, o Caipe identificou alguns princípios para auxiliar no desenvolvimento da EIP.

Esses princípios se baseiam no grau de satisfação do atendimento e do tratamento dispensado aos pacientes; incentivam os profissionais a aprenderem juntos e entre si; melhoram as relações e as atitudes diante da prática profissional; e auxiliam a equipe na conquista do apoio mútuo e da responsabilidade conjunta ${ }^{4}$.

O serviço de saúde, ao atender a comunidade de acordo com suas necessidades, propõe medidas que articulem os diferentes profissionais quanto a uma atuação coletiva. A prática colaborativa facilita a relação entre eles; permite que cada profissão tenha não só uma compreensão mais profunda de seu próprio desempenho, mas também complemente e potencialize o de outros profissionais. Todos são vistos como semelhantes, mesmo que as diferenças de poder, a posição ou o status no trabalho ${ }^{4}$ sejam patentes.

De acordo com Pinho ${ }^{24}$, a incorporação da especialização, somada à competência e habilidade técnica agregada a um conhecimento mais amplo, tem ajudado os profissionais de saúde a superar desafios e a romper concepções de saúde fragmentadas e isoladas. Esses profissionais passam a ter uma percepção mais abrangente, dinâmica, crítica, reflexiva e integrada à saúde.

As necessidades de saúde da população se constituem, ainda, em um desafio para aqueles centros de formação da área da saúde carentes de cenários de aprendizagem, nos quais seja desenvolvida a prática interprofissiona ${ }^{20}$. Desse modo, a RMS permite que o residente permaneça durante dois anos em um espaço, no qual lida, constantemente, com as relações interpessoais, habilidades e atitudes que garantem a articulação do seu trabalho com as necessidades de saúde. Para McNair ${ }^{25}$, a prática colaborativa da EIP promove a comunicação eficaz, a colaboração e o trabalho entre os membros da equipe e aprimora a assistência ao cuidado de saúde.

Em seu estudo, Olson ${ }^{26}$ explica que a aprendizagem, em cenários de prática com paciente, tornase mais eficaz quando compartilhada com pequenos grupos de profissionais, em que as atitudes e o conhecimento de seus papéis são mais bem desenvolvidos e aplicados na equipe interprofissional.

A categoria identificação das necessidades dos pacientes e da comunidade ressalta a importância do trabalho de equipe na atenção integral ao usuário. Nessa perspectiva, a equipe revisa seu planejamento referente às ações de saúde e à responsabilização pelo cuidado.

Ao realizar uma visita domiciliar com outros profissionais consigo visualizar as diversas demandas do paciente (nutricional, fono, fisioterapeuta) [...]. (E1)

Estas discussões promovem práticas interdisciplinares com foco na integralidade do cuidado. As ações são sempre de corresponsabilidade entre os membros da equipe. (E19)

É importante destacar que, para os autores Machado et al. ${ }^{27}$, a integralidade pode ser vista como uma dimensão do cuidado. Os profissionais de saúde direcionam o seu interesse no resultado terapêutico do cuidado em saúde para além da dimensão do sujeito doente, sob uma perspectiva que engloba estados subjetivos de satisfação.

Cecílio ${ }^{28}$ afirma que as necessidades de saúde ampliam a linha de cuidado em saúde que passam a priorizar a intervenção e a prática. Existe a presença de laços afetivos entre o usuário e a equipe que reflete no vínculo e na confiança, o que é extremamente positivo para o cuidado em saúde e aderência ao tratamento.

Na residência, o trabalho de equipe viabiliza ações conjuntas em prol do paciente em uma relação de reciprocidade entre o trabalho e a interação. Para Peduzzi ${ }^{29}$, é importante que a comunicação entre profissionais faça parte do exercício cotidiano de trabalho e permita articular inúmeras ações na equipe, no serviço e na rede de atenção.

A categoria organização do serviço de saúde revela a importância das instituições de ensino e dos serviços de incentivar a EIP na equipe. Os residentes valorizam e reconhecem esse apoio. 
A residência multiprofissional permite o convívio com os profissionais das outras áreas, porém a cultura de uma prática colaborativa precisa ser desenvolvida e cultivada diariamente, fato que depende da estrutura e organização do serviço, nem sempre favorecida na prática. (E26)

As instituições, os gestores e os serviços devem se comprometer com a eficácia da EIP9 em forma de um apoio organizacional que deve incluir: liderança na condução do processo; conhecimento; experiência com essa abordagem; e a formação de um corpo docente proativo que incentive os estudantes.

O planejamento e a implementação da EIP são etapas árduas que encontram barreiras organizacionais quanto ao número de estudantes, requisitos de acreditação profissional, currículo inflexível e a logística para os locais de aprendizado. Para os autores Batista e Batista ${ }^{9}$, é necessário o apoio das instituições para que os profissionais de saúde participem dos programas e tornem possíveis as mudanças para a eficácia interprofissional.

Os responsáveis pelo ensino e pela prática de atuação precisam colocar as necessidades dos usuários no centro de seus interesses e propiciar o espaço para a EIP4. Ademais, a participação ativa e o envolvimento dos responsáveis, com respostas adequadas às necessidades, implicam em um planejamento e em uma avaliação dos projetos de implantação da EIP, nos cenários de ensino.

Ao se pautar no trabalho de equipe, a residência encontra dificuldades no serviço e na percepção dos outros profissionais, uma vez que a atuação integrada é uma prática que depende das características dos serviços.

[...] quando nos deparamos com a prática somos bloqueados pela demanda de serviço, pela falta de percepção dos profissionais do próprio hospital sobre a residência e a falta de estrutura da instituição. A residência produz a multidisciplinaridade que já é vivenciada no hospital, não conseguimos realizar mudanças na forma de atuação existente. (E16)

A prática e as condições de trabalho precisam ser estruturadas a fim de fornecer o aporte necessário ao residente em sua atuação, como componente da equipe e com os demais profissionais inseridos nos serviços.

A cooperação intersetorial e multiprofissional deve ser o alicerce para o trabalho, atrelado ao embasamento proporcionado pela formação acadêmica. Entretanto, na prática seu funcionamento é muitas vezes distorcido. Os profissionais residentes pela demanda do serviço assumem como mão de obra barata. (E23)

É pertinente que as IES e os PRMS formem profissionais com uma escuta qualificada e um olhar para além do processo saúde-doença que estejam atentos para o cuidado, voltado a uma perspectiva de transformação da rotina do trabalho de equipe. Ademais, formar profissionais exige uma atenção para um aprendizado mais crítico e reflexivo, com uma relação entre a teoria e a prática. Esses seriam alguns pressupostos para a formação, além da qualificação do corpo docente.

Ceccim ${ }^{30}$ destaca que a insuficiência dos treinamentos formais e teóricos emerge um movimento entre os pesquisadores da área da saúde que expressam a necessidade de formação que incluam o compartilhamento de saberes e de experiências no desenvolvimento de competências profissionais, com o uso de metodologias ativas de ensino-aprendizagem nos processos de educação permanente em saúde (EPS).

Essas instâncias formativas podem superar desafios nos cenários, nos quais as equipes atuam e produzam um efeito negativo no desempenho.

[...] na prática vivenciamos campos de estágio diferentes e desenvolvemos trabalhos a parte do serviço por muitas vezes sem que os funcionários do setor estejam cientes de nosso trabalho. 
O ideal da RMS deveria preparar o residente para uma prática colaborativa; no dia a dia isso não acontece. O residente acaba sendo engolido pela dinâmica da unidade em que está com profissionais despreparados para lidar com a prática multiprofissional e não consegue transformar o que é ideia na realidade. (E64)

Para consolidar a EIP e a prática colaborativa, Peduzzi ${ }^{i 1}$ afirma que é preciso ter o conhecimento das resistências, encontradas ao longo do percurso. Caso contrário, corre-se o risco de reforçar os conceitos e os modelos tradicionais, bem como se deparar com uma abordagem biomédica inflexível e uma atuação profissional isolada e autônoma no campo da saúde que é complexo, interprofissional e interdisciplinar.

Câmara et al..$^{32}$ afirmam que a EIP necessita se estabelecer mediante o apoio institucional das universidades e se fortalecer com as políticas indutoras de formação para tornar possível uma ampliação do diálogo com todas as profissões da saúde, bem como manter uma efetiva articulação entre os gestores dos serviços em todos os níveis de governo, universidades, profissionais, órgãos reguladores das profissões, usuários e população, a fim de superar os modelos predominantes de educação e prática uniprofissional que não correspondem às reais necessidades de saúde da população.

\section{Considerações finais}

A RMS se constitui em uma ação estratégica para transformar a organização dos serviços, o processo de formação, as ações de saúde e as práticas pedagógicas que, por sua vez, resultaram em novos processos de trabalho, articulados com as instituições formadoras e com o sistema de saúde.

Os PRMS e os PP analisados evidenciam potencialidades que explicitam ações de trabalho que conduzem à responsabilização das equipes no que tange ao cuidado e à capacidade de resolver os problemas da saúde. Essas equipes devem priorizar, conjuntamente, a elaboração de planos de cuidado. Esse tipo de atitude favorece a confiança mútua e a colaboração entre os profissionais na busca da qualidade do atendimento. É fundamental para o trabalho de equipe nos PRMS a promoção de seus avanços na conceituação dos seus princípios e no detalhamento das estratégias a serem implementadas na perspectiva da EIP.

A percepção sobre o desenvolvimento de competências para a prática colaborativa evidenciou a satisfação dos residentes no que se refere ao trabalho de equipe, ao respeito mútuo, ao reconhecimento do seu papel e do outro profissional, o que permite a troca de experiências, de saberes e de corresponsabilidade na rotina de trabalho da equipe. O momento de compartilhar as ações a favor do paciente são oportunidades excelentes de integração e aprendizado.

Entre os desafios encontrados estão a condição de trabalho pela expressiva demanda e a visão especializada que dificulta o atendimento na perspectiva da integralidade do cuidado. Nesse modelo, cabe aprimorar as estratégias de qualidade de atendimento.

Os PRMS visam promover mudanças na qualificação profissional e fomentar a integração ensinoserviço, desde que assegurem a organização dos serviços e a integralidade como eixo orientador das práticas da atenção.

A EIP incentiva novas práticas e ações em saúde no processo de trabalho, no currículo e na formação do profissional. De acordo com a visão dos residentes, identificam-se as potencialidades e as fragilidades tanto das instituições de formação quanto dos próprios profissionais. Esse fato demonstra a necessidade de um debate acerca da colaboração na construção das ações de saúde para contribuir no desenvolvimento de estratégias entre as equipes no que se refere ao trabalho de equipe interprofissional.

A EIP é uma necessidade que se constitui em um desafio na relação dos profissionais da saúde nos processos de formação, nos vínculos e na comunicação. 


\section{Colaboradores}

Isis Alexandrina Casanova participou da concepção e do desenho do estudo; da coleta, análise e interpretação dos dados; e da escrita, revisão e aprovação final do trabalho. Lídia Ruiz Moreno e Nildo Alves Batista participaram da análise e interpretação dos dados; e da escrita, revisão e aprovação final do trabalho.

\section{Referências}

1. Lei $n^{\circ} 11.129$, de 30 de Junho de 2005. Institui o Programa Nacional de Inclusão de Jovens - Pró jovem: cria o Conselho Nacional da Juventude (CNJ) e a Secretaria Nacional da Juventude; altera as Leis $n^{\circ} 10.683$, de 28 de maio de 2003, e 10.429, de 24 de abril de 2002; e dá outras providências. Diário Oficial da União. 30 jun. 2005 [acesso 4 Jun 2013]. Disponível em: http://www.planalto.gov.br/ccivil_03/_ato2004-2006/2005/lei/ I11129.htm.

2. Salvador AS, Medeiros CS, Cavalcanti PB, Carvalho RN. Construindo a multiprofissionalidade: um olhar sobre a residência multiprofissional em saúde da família e comunidade. Rev Bras Cienc Saude. 2011; 15(3):329-38.

3. Organização Mundial da Saúde. Marco para ação em educação interprofissional e prática colaborativa [Internet]. Genebra: OMS; 2010 [acesso 2 Maio 2012]. Disponível em: http://new.paho.org/bra/images/stories/documentos/marco_para_acao.pdf\% 20.

4. Centre for the Advancement of Interprofessional Education. Defining IPE [Internet]. Fareham: CAIPE; 2002 [acesso 4 Jun 2013]. Disponível em: http://www.caipe.org.uk/ resources/defining-ipe/.

5. Canadian Interprofessional Health Collaborative (CIHC) [Internet]. [acesso 4 Jun 2013]. Disponível em: http://www.cihc.ca/.

6. Furtado JP. Equipes de referência: arranjo institucional para potencializar a colaboração entre disciplinas e profissões. Interface (Botucatu). 2007; 11(22):239-55.

7. Rossit RAS, Batista SHS, Batista NA. Formação para a integralidade no cuidado: potencialidades de um projeto interprofissional. Rev Int Humanidades Med [Internet]. 2014 [acesso 4 Jun 2013]; 3(1):55-64. Disponível em: http://www2.unifesp.br/centros/ cehfi/documentos/revista_hum_med_vol3_num1_2014.pdf.

8. Barr $\mathrm{H}$. Interprofessional education: the genesis of a global movement [Internet]. London: CAIPE; 2015 [acesso 4 Ago 2015]. Disponível em: https://www.caipe.org/ download/barr-h-2015-interprofessional-education-genesis-global-movement/.

9. Batista NA, Batista SHSS. Educação interprofissional na formação em saúde: tecendo redes de práticas e saberes. Interface (Botucatu). 2016; 20(56):202-4.

10. Minayo MCS. O desafio do conhecimento: pesquisa qualitativa em saúde. 12a ed. Rio de Janeiro: Hucitec; 2008.

11. Lakatos EM, Marconi MA. Fundamentos de metodologia científica. 6a ed. São Paulo: Atlas; 2005.

12. Ministério da Educação (BR). Instituições credenciadas [Internet]. Brasília: Ministério da Educação; 2013 [acesso 13 Out 2013]. Disponível em: http://portal.mec.gov.br/ instituicoes-credenciadas-sp-1781541355.

13. Bardin L. Análise de conteúdo. Lisboa: Edições 70; 2009.

14. Perego MG. Aprendizagens compartilhadas na residência multiprofissional em saúde [dissertação]. Santos(SP): Universidade Federal de São Paulo; 2015.

15. Lakatos EM, Marconi MA. Técnicas de pesquisa. 3a ed. São Paulo: Atlas; 1996. 
16. Moraes SG, Justino ML, Ferreira BJ, Barbosa EP, Bruno LF, Pereira LAV. Development and validation of strategy to assess teaching methods. In: Nata RV, editor. Progress in education [Internet]. New York: Nova Science Publishers; 2012 [acesso 13 Out 2013]. v. 28, p. 81-107. Disponível em: https://www.novapublishers.com/catalog/product_info. php?products_id $=35562$.

17. Aguilar-da-Silva RH, Scapin LT, Batista NA. Avaliação da formação interprofissional no ensino superior em saúde: aspectos da colaboração e do trabalho em equipe. Avaliação (Campinas). 2011; 16(1):165-82.

18. Ceccim RB. Equipe de saúde: a perspectiva entre-disciplinar na produção dos atos terapêuticos. In: Pinheiro R, Mattos RA, organizadores. Cuidado: as fronteiras da integralidade. Rio de Janeiro: IMS/UERJ; 2004. p. 259-78.

19. D'Amour D, Goulet L, Labadie JF, Martín-Rodriguez L, Pineault R. A model and typology of collaboration between professionals in healthcare organizations. BMC Health Serv Res. 2008; 8(1):188. doi: 10.1186/1472-6963-8-188.

20. Motta LB, Pacheco LC. Integrating medical and health multiprofessional residency programs: the experience in buildingan interprofessional curriculum for health professionals in Brazil. Educ Health. 2014; 27(1):83-8. doi: 10.4103/1357-6283.134331.

21. Silva HAR. Educação interprofissional na graduação em saúde: aspectos avaliativos da implantação na Faculdade de Medicina de Marília (Famema). Educar Rev. 2011; (39):159-75. doi: 10.1590/S0104-40602011000100011.

22. Silva JMA. Educação interprofissional em saúde e enfermagem no contexto da atenção primária [tese]. São Paulo: Escola Paulista de Enfermagem, Universidade de São Paulo; 2014.

23. Ellery ELA, Pontes JSR, Loiola FA. Comunidade de prática enquanto modo coletivo de aprendizagem e desenvolvimento de práticas e saberes na estratégia de saúde da família: um estudo teórico. Rev Bras Promoc Saude. 2012; 25(2):104-12. doi: 10.5020/2251.

24. Pinho MCG. Trabalho em equipe de saúde: limites e possibilidades de atuação eficaz. Cienc Cogn. 2006; 8:68-87.

25. McNair RP. The case for educating health care students in professionalism as the core content of interprofessional education. Med Educ. 2005; 39(5):456-64. doi: 10.1111/j.1365-2929.2005.02116.x.

26. Olson R, Bialocerkowski A. Interprofessional education in allied health: a systematic review. Med Educ. 2014; 48(3):236-46. doi: 10.1111/medu.12290.

27. Machado MFAS, Monteiro EMLM, Queiroz DT, Vieira NFC, Barroso MGT. Integralidade, formação de saúde, educação em saúde e as propostas do SUS - uma revisão conceitual. Cienc Saude Colet. 2007; 12(2):335-42. doi: 10.1590/S141381232007000200009.

28. Cecílio LCO. As necessidades de saúde como conceito estruturante na luta pela integralidade e equidade na atuação em saúde. In Pinheiro R, Mattos RA, organizadores. Os sentidos da integralidade na atenção e no cuidado à saúde. Rio de Janeiro: UERJ, IMS, ABRASCO; 2006. p. 117-30.

29. Peduzzi M. Equipe multiprofissional de saúde: conceito e tipologia. Rev Saude Publica. 2001; 35(1):103-9. doi: 10.1590/S0034-89102001000100016.

30. Ceccim RBB. Desenvolvimento ou competências no trabalho em saúde: educação, áreas do conhecimento e profissões no caso da saúde. Tempus. 2012; 6(2):253-77. doi: 10.18569/tempus.v6i2.1128.

31. Peduzzi M. O SUS é interprofissional. Interface (Botucatu). 2016; 20(56):199-201. doi: 10.1590/1807-57622015.0383. 
32. Câmara AMCS, Cyrino AP, Cyrino EG, Azevedo GD, Costa MV, Bellini MIB, et al. Educação interprofissional no Brasil: construindo redes formativas de educação e trabalho em saúde. Interface (Botucatu). 2016; 20(56):5-8. doi: 10.1590/1807-57622015.0700.

Casanova IA, Batista NA, Moreno LR. La educación Interprofesional y la práctica compartida en programas de residencia multiprofesional de Salud. Interface (Botucatu). 2018; 22(Supl.1):1325-37.

El objetivo del artículo es analizar los principios, concepciones y prácticas de la Educación Interprofesional, con énfasis en la práctica compartida en programas de residencia multiprofesional de Salud (PRMS), en las IES del Estado de São Paulo, Brasil. Se realizaron: análisis documental de los Proyectos Pedagógicos (PP), aplicación de instrumento tipo Likert y preguntas abiertas a 76 residentes de 13 PRMS. El análisis temático de los PP muestra: énfasis en que el equipo asuma la responsabilidad ante los casos clínicos y planes de cuidado; centralización en las necesidades del paciente y articulación entre enseñanzaservicio. Los resultados del Likert ponen en evidencia: satisfacción de los residentes con la formación para práctica colaborativa. En la perspectiva de los residentes, los PRMS amplían y mejoran los resultados de salud; favorecen la actuación centrada en el paciente; identificación de las necesidades de salud; compartición de prácticas y procedimientos. Por lo tanto, los PRMS son importantes en el escenario de consolidación del SUS.

Palabras clave: Formación de recursos humanos. Internado y residencia. Educación interprofesional. 
